\title{
Estudio de la influencia de la microestructura sobre la deformabilidad en caliente de un acero inoxidable dúplex
}

\author{
A. Iza-Mendia ${ }^{(*)}$, A. Piñol-Juez ${ }^{(*)}$ e I. Gutiérrez ${ }^{(*)}$ \\ Resumen El comportamiento frente al conformado en caliente de la ferrita y de la austenita, en una estructura \\ dúplex, es muy diferente al que presentan ambas fases por separado en los aceros monofásicos auste- \\ níticos o ferríticos. A ello contribuyen, entre otros, la distribución espacial de las fases con respecto a \\ la deformación impuesta, la codeformación de dos fases, con propiedades mecánicas muy diferentes, \\ y la naturaleza de la intercara. En el presente trabajo se analiza la influencia de estos factores en la \\ formación de daño bajo condiciones de deformación en caliente. \\ Palabras clave: Acero inoxidable austenoferrítico: Ductilidad en caliente. Deslizamiento de la \\ intercara $\delta / \gamma$.

\section{Study of the effect of the microstructure on the hot deformability of a duplex stainless steel}

\begin{abstract}
The complexity of the hot deformation behaviour of the ferrite and austenite in a duplex structure is increased as compared with that of single phase ferritic or austenitic steels. Important factors are: the spatial phase distribution with respect to the direction of the imposed deformation, the codeformation of both phases having considerably different mechanical properties, and the nature of the interface between austenite and ferrite. In the present study, the influence of these factors on the crack formation during the hot deformation is analyzed.
\end{abstract}

Keywords: Duplex stainless steel. Hot ductility. Sliding at the $\delta / \gamma$ interface.

\section{INTRODUCCIÓN}

Los aceros inoxidables dúplex austenoferríticos son materiales que presentan una óptima combinación de propiedades mecánicas y de resistencia a la corrosión en comparación con los aceros inoxidables convencionales austeníticos o ferríticos. Sin embargo, el procesamiento industrial en caliente de estos materiales constituye una etapa crítica que conlleva, en numerosos casos, la formación de grietas o un acabado superficial inaceptable.

En el presente trabajo se ha estudiado el comportamiento durante la deformación en caliente, en modo torsión, de un acero inoxidable dúplex con

(*) Centro de Estudios e Investigaciones Técnicas (CEIT) y Escuela Superior de Ingenieros Industriales (ESII), Univ. de Navarra. $\mathrm{P}^{\circ}$. de Manuel Lardizabal 15. 20009-DonostiaSan Sebastián (España). diferentes estructuras procedentes de colada y de laminación en caliente.

\section{MÉTODO EXPERIMENTAL}

La composición química, expresada en tanto por ciento en masa, del acero inoxidable dúplex estudiado en el presente trabajo es la siguiente: $23 \mathrm{Cr}-$ 4.8Ni-1.3Mn-0.22Mo-0.025C-0.095N. Este acero fue suministrado por CSM (Roma) en dos condiciones diferentes: en estado bruto de colada, y en estado laminado con una reducción del $77 \%$. La fracción volumétrica de austenita de ambas muestras resultó ser del 65 y $50 \%$, respectivamente.

Se realizaron ensayos de torsión a 1.000, 1.100 y $1.200{ }^{\circ} \mathrm{C}$ a una velocidad de deformación de $1 \mathrm{~s}^{-1}$, tras $1 \mathrm{~h}$ de mantenimiento antes del ensayo. Después de deformadas, las probetas se enfriaron en agua. La observación microestructural se realizó en 
el radio efectivo de las probetas de torsión (1). La microscopía electrónica de barrido se llevó a cabo mediante los microscopios electrónicos Philips XL30 SEM y Philips CM12 STEM.

\section{RESULTADOS Y DISCUSIÓN}

En la figura 1 se muestra la microestructura del acero dúplex en los estados bruto de colada y laminado. El material bruto de colada posee una estructura dendrítica de austenita en una matriz ferrítica. Las dos fases presentan una estructura de tamaño de grano grosero, estando la austenita constituida por monocristales (Fig. 1a y 1b). En el material laminado, las fases constituyentes están orientadas en la dirección de laminación. Ambas fases poseen una estructura totalmente recristalizada como consecuencia del tratamiento térmico previo al ensayo (Fig. 1c y 1d). La diferencia entre el material bruto de colada y el laminado reside en la distribución espacial de las fases, así como en el tipo de intercara existente entre la austenita y la ferrita. Debido a la composición del presente acero la austenita se forma a partir de la ferrita delta, tras la solidificación, transformación que conlleva la existencia de una relación de orientación a través de la intercara ferrita/austenita del tipo Kurdjumov-Sach (K-S) en
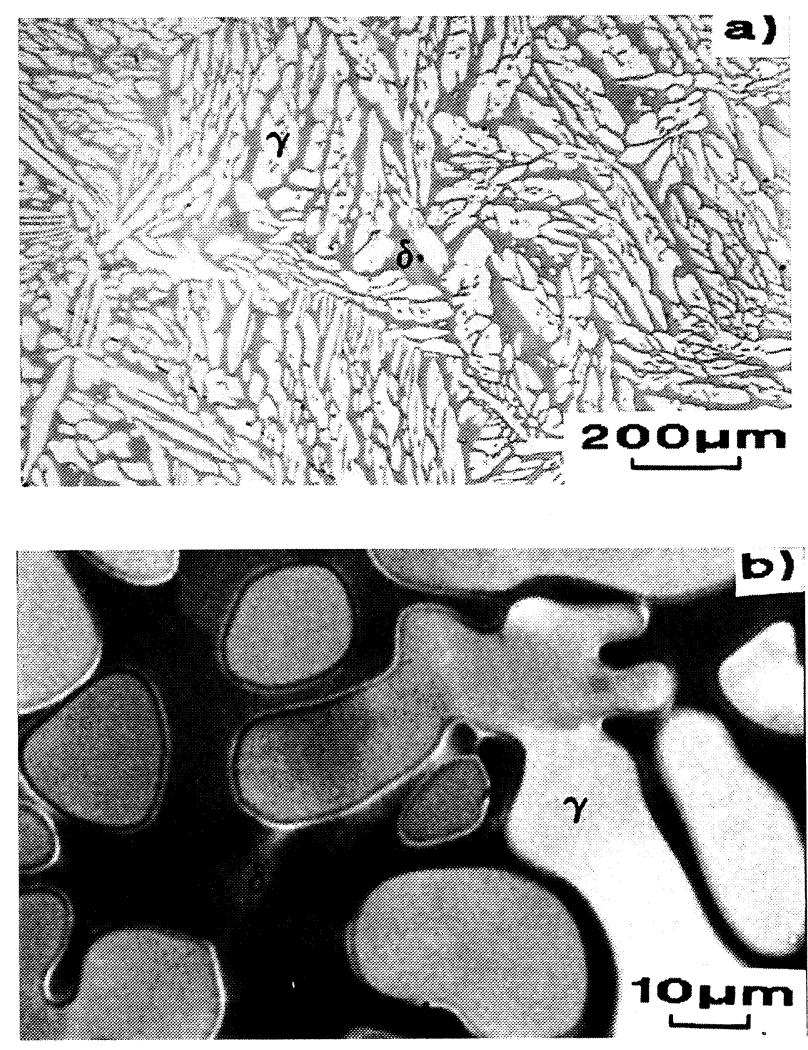

el material bruto de colada. Esta observación concuerda con el trabajo de otros autores (2). Sin embargo, los estudios realizados demuestran que esta relación se pierde en el material laminado como consecuencia de la deformación y/o recristalización (3).

En la figura 2 se muestran las curvas tensióndeformación del material bruto de colada y del laminado para $T=1.000{ }^{\circ} \mathrm{C}$ y $\varepsilon=1 \mathrm{~s}^{-1}$. En estado bruto de colada, la tensión de fluencia alcanza un máximo para una deformación de $\varepsilon=0,15$. A partir de ese momento, la tensión decrece monótonamente conforme la deformación progresa. En el material laminado a bajas deformaciones, el endurecimiento resulta ser de tipo parabólico hasta alcanzar el valor de tensión $\sigma_{0}$. Seguidamente, el endurecimiento es prácticamente lineal, alcanzándose un pico de tensión $\sigma_{\mathrm{p}}$ para una deformación de 0,8 , a partir de la cual la tensión decrece continuamente hasta fractura.

En la figura 3 se muestran las probetas de torsión llevadas hasta deformaciones semejantes a diferentes temperaturas. De su observación, se desprende que la probeta deformada a $1.000^{\circ} \mathrm{C}$ presenta un elevado número de grietas en la superficie, que prosiguen su avance a través de toda la sección. Estas grietas aparecen antes de la fractura final del material y se concentran en la intercara ferrita/
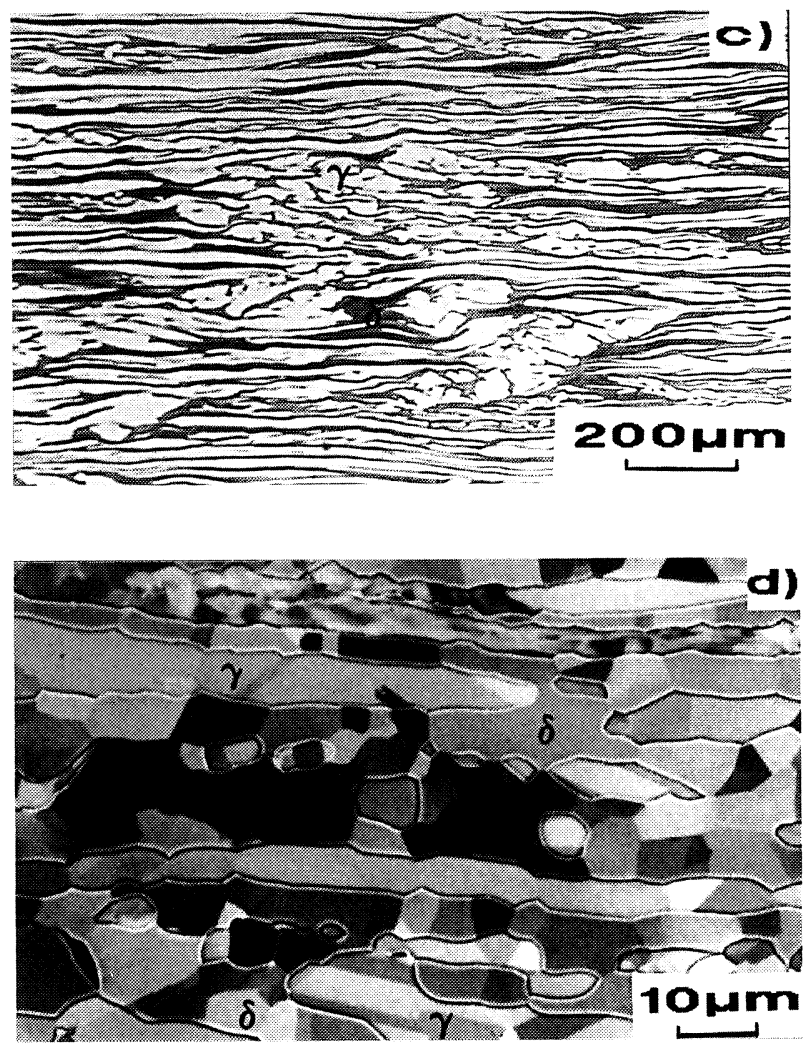

FIg. 1.- Micrografías de óptico y SEM a) y b) bruto de colada. c) y d) material laminado. FIG. 1.- Optical and SEM micrographs a) and b) as-cast.c) and d) wrought condition. 


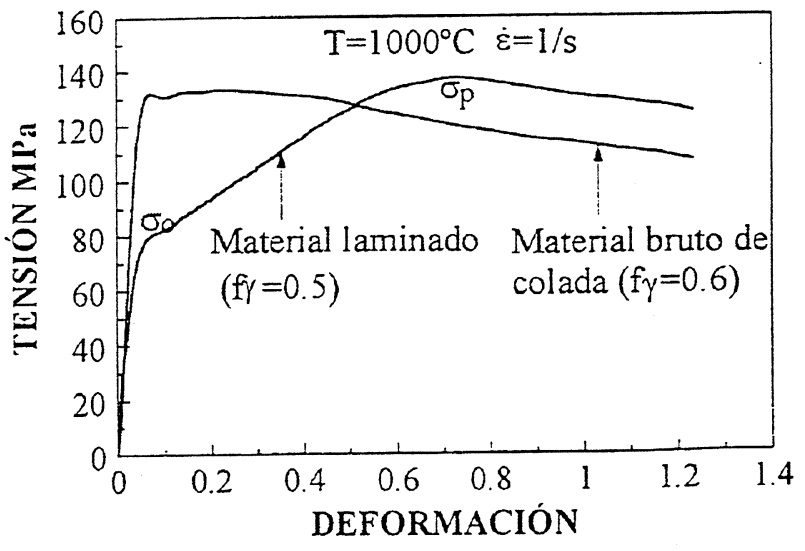

Fig. 2.- Curvas tensión-deformación del dúplex deformado en torsión a $1.000{ }^{\circ} \mathrm{C}$.

FIG. 2.- Stress strain curves of the DSS deformed by torsion at $1,000^{\circ} \mathrm{C}$.
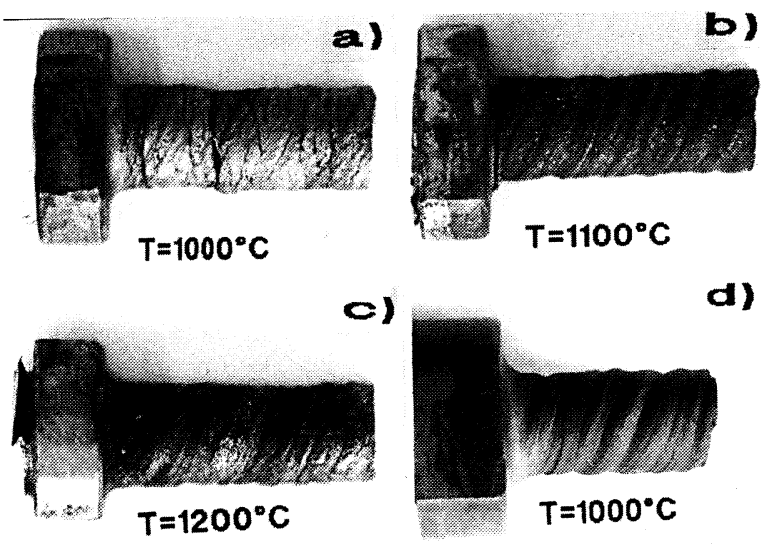

FIG. 3.- Probetas de torsión tras una deformación de $\varepsilon=1,6$ a $\varepsilon=1 \mathrm{~s}^{-1}$, del material : a) b) y c) bruto de colada. d) laminado.

FIG. 3.- Hot torsion specimens after deformation to $\varepsilon=1.6$ at $\varepsilon=1 \mathrm{~s}^{-1}$, corresponding to a) b) and c) as-cast.d) as-wrought material.

austenita (Fig. 4). En las muestras deformadas a 1.100 y $1.200^{\circ} \mathrm{C}$, se observa un menor número de grietas, que son únicamente superficiales. Por el contrario, no se observan grietas en la probeta del material laminado torsionada a $1.000^{\circ} \mathrm{C}$. Ello significa que la ductilidad del material no sólo depende de la ductilidad de las fases constituyentes $\delta$ y $\gamma$, sino también de la distribución de ambas en el material y probablemente de la naturaleza de la intercara entre ellas.

Puede verse en las estructuras deformadas que algunos granos de austenita parecen no haberse deformado de la misma manera que el resto del material, incluso a grandes deformaciones (Fig. 1c); y no evolucionan hacia una configuración alargada, manteniendo por el contrario una geometría más

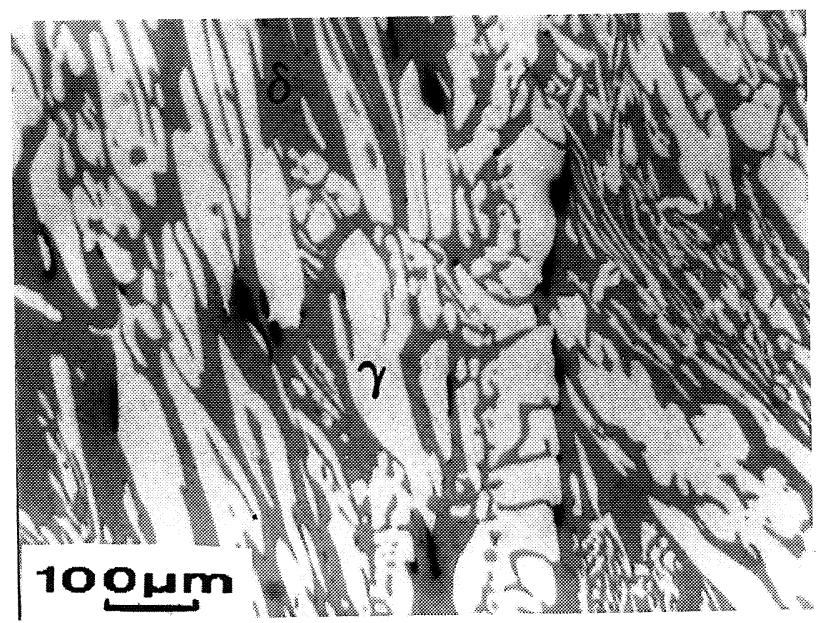

Fig. 4.- Micrografía óptica del material bruto de colada deformado en torsión a $1.000{ }^{\circ} \mathrm{C}$ y mostrando grietas que avanzan paralelamente a la intercara $\delta / \gamma$.

FIG. 4.- Optical micrograph of the as-cast material deformed by hot torsion at $1,000{ }^{\circ} \mathrm{C}$, showing cracks running parallel to the $\delta / \gamma$ interface.

esférica. Estos granos de austenita aparecen como inclusiones, mientras que el resto del material fluye alrededor de ellos. En la figura 5 se muestra la microestructura de deformación del material bruto de colada a $1.000^{\circ} \mathrm{C}$, correspondiente posiblemente a una zona de este tipo, como lo demuestra la forma no alargada del grano 1 de austenita, la ausencia casi total de estructura fina en su interior y el mantenimiento todavía de la relación de orientación de $K-S$ a través de su intercara con la ferrita. Es de notar la presencia de grietas en la intercara $\delta / \gamma$ correspondiente al citado grano, que pueden interpretarse como la manera de relajar las tensiones impuestas por la intercara semicoherente.

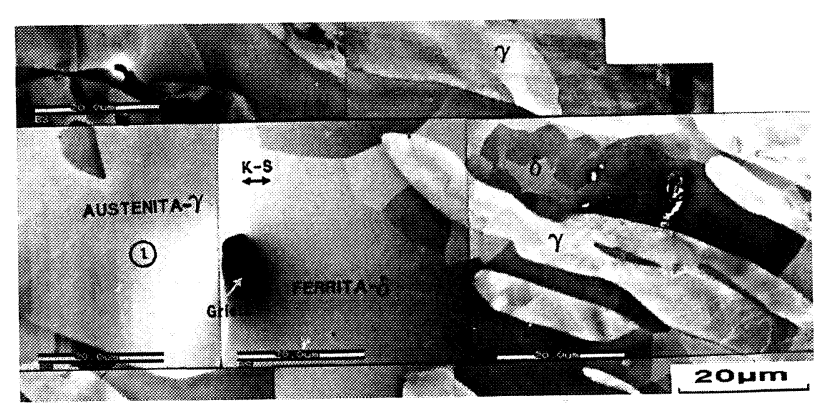

FIG. 5.- Imagen de SEM obtenida mediante contraste de electrones primarios del material deformado a $1.000^{\circ} \mathrm{C}$, llevado hasta $\varepsilon=1,6$ a $\varepsilon=1 \mathrm{~s}^{-1}$.

FIG. 5.- SEM channelling contrast back scattered electron image obtained on the as-cast material deformed at $1,000^{\circ} \mathrm{C}$ to a $\varepsilon=1,6$ at $\varepsilon=1 \mathrm{~s}^{-1}$. 
El diferente comportamiento mecánico de las fases ferrita $(\delta)$ y austenita $(\gamma)$ conduce a que la fase más blanda a alta temperatura, $\delta$, se deforme plásticamente en mayor medida que la fase más dura $\gamma$ (4). En un material bifásico como el acero inoxidable dúplex, la diferencia en resistencia de ambas fases puede forzar a un deslizamiento en la intercara entre austenita y ferrita, mecanismo no observado en los aceros monofásicos (5).

Se han realizado ensayos de torsión sobre probetas pulidas y marcadas. La observación de la superficie tras el ensayo mediante SEM pone de manifiesto (Fig. 6) la existencia de cierto deslizamiento en la intercara $\delta / \gamma$, tal y como demuestra la discontinuidad de las rayas, inicialmente rectas, trazadas a través de los granos de austenita y ferrita. Como resultado del deslizamiento, se forman grietas en el bruto de colada, que se indican mediante flechas en la micrografía de la figura 6a. No así en el laminado (Fig. 6b). Son claras las diferencias observadas entre el comportamiento de ambos materiales. En el bruto de colada, las trazas mantienen su continuidad a lo largo de grandes distancias y el desplazamiento se concentra, al menos para deformaciones macroscópicas relativamente bajas, en zonas como las que se muestran en la figura $6 \mathrm{a}$, produciendo grietas importantes a lo largo de las intercaras ferrita/austenita. Estas zonas parecen coincidir con las paredes celulares de la estructura de solidificación. Por el contrario, en el material previamente laminado, la propia disposición de las fases y la naturaleza

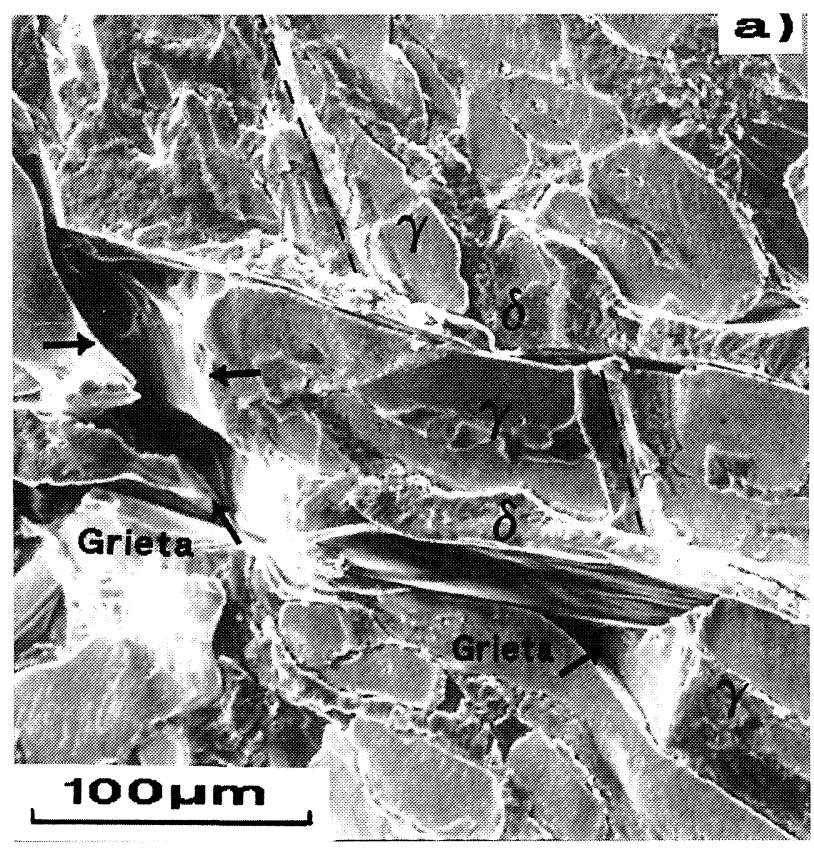

incoherente de la intercara entre ellas pueden ser las responsables de que el deslizamiento se distribuya más homogéneamente en todas las intercaras $\delta / \gamma, y$ sin las concentraciones comentadas anteriormente (Fig. 6b). Así, la estructura dúplex se acomoda mejor a la deformación macroscópica impuesta y no se producen grietas.

\section{CONCLUSIONES}

La diferente distribución de las fases $\delta$ y $\gamma$ en el material bruto de colada y el material laminado, así como la naturaleza de la intercara austenoferrítica parecen determinar la deformabilidad en caliente del acero inoxidable dúplex.

El daño originado en la intercara ferrita/austenita en el material bruto de colada parece tener, al menos, dos causas: la resistencia de algunos granos de austenita a deformarse y la concentración del deslizamiento sobre las paredes celulares de la estructura de solidificación. En el material previamente laminado, el deslizamiento se distribuye más homogéneamente y se evita la formación de grietas.

\section{Agradecimiento}

Parte de este trabajo se ha desarrollado en el marco de un proyecto CECA ${ }^{\circ}$. 7210-EC/932, en colaboración con el Dr. J. Bianchi, del CSM

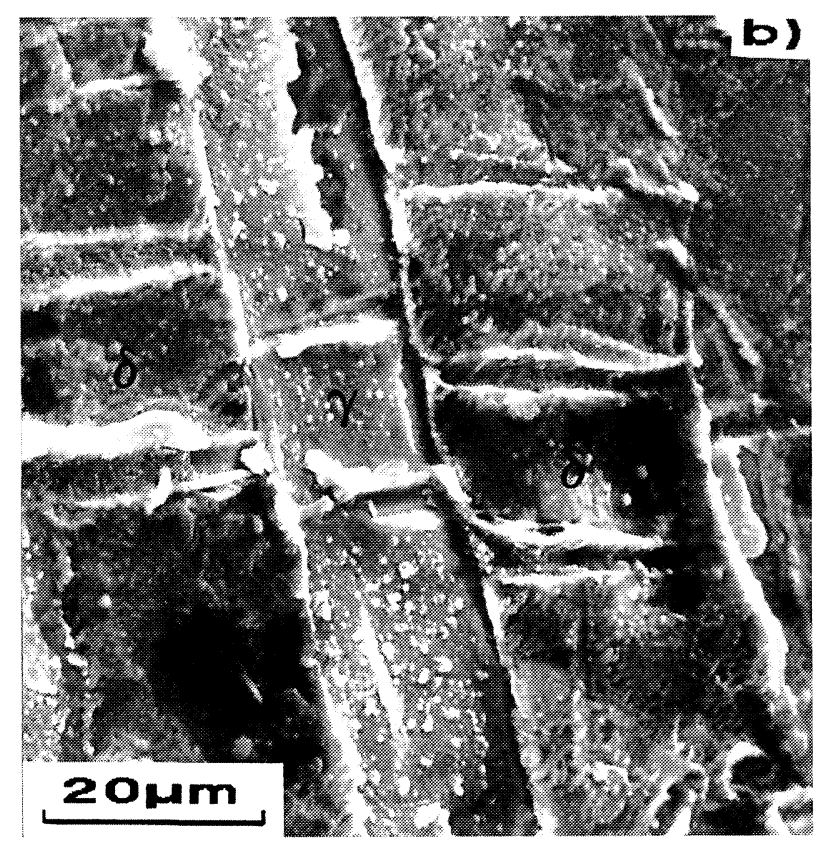

FIG. 6.- Deslizamiento de la intercara $\delta / \gamma$ a) con formación de grietas en el material bruto de colada y b) ausencia de daño en el material laminado.

FIG. 6. $-\delta / \gamma$ interphase sliding a) with cracks appearing in the as-cast material and $b$ ) with no damage in the as-wrought material. 
(Roma), y financiado por la Comunidad Europea. A. I-M y A. P-J agradecen al Gobierno Vasco la concesión de becas para la formación de investigadores. Los autores quieren dedicar este artículo al Prof. J.J. Urcola, que trabajó en el proyecto antes de su muerte prematura, y al que agradecen sus enseñanzas, su entusiasmo contagioso y su amistad.

\section{REFERENCIAS}

(1) Barraclough, D.R., Whittaker, H.J., Nair, K.D. y Sellars, C.M. J. Testing and Evaluation, 1 (3), 1973 : 220-226.
(2) Solomon, H.D. y Devine, T.M. Proc. Duplex Stainless Steels, Ed. R.A. Lula ASM Publ., St.Louis, (Mo. EE.UU.) 1983: 693-756.

(3) Iza-Mendia, A. Tesis doctoral, Univ. de Navarra, Donostia-San Sebastián. En curso.

(4) Chandra, T., Dunne, D.P. y Campbell, P. Proc. ICSMA 7, Eds McQueen et al., 1986: 947-952.

(5) Al-Jouni, F.E. y Sellars, C.M. Deformation of MultiPhase and Particle Containing Materials, Risø Conf., B. Sørensen et al. ed., Roskilde (Dinamarca), 1983: 131-137. 